\title{
Maternal and fetal benefits of DHA supplementation during pregnancy
}

\author{
Corinne Hubinont ${ }^{1 *}$ and Thomas Savoye ${ }^{2}$ \\ ${ }^{1}$ Saint Luc University Hospital, 10 avenue Hippocrate, B-1200 Brussels, Belgium \\ ${ }^{2}$ Independent consultant in medical affairs, Van Couwenhovelaan 7, 3020 Herent, Belgium
}

\begin{abstract}
During pregnancy, the benefits of long-chain n-3 fatty acids, mainly DHA supplementation has been reported with controversial results. The aim of this review paper is to clarify the role of DHA on maternal and fetal outcomes and to discuss supplementation.

Low maternal concentrations of DHA are associated with an increased incidence of preeclampsia, gestational diabetes, postpartum depression, preterm labour and intrauterine fetal growth restriction (IUGR).

The effect of a preventive supplementation on these complications is not clearly demonstrated . Some authors report an improved outcome but recent Cochrane database systematic reviews and meta-analysis are not conclusive.

DHA supplementation reduces significantly the effects of prenatal stress and the perinatal mortality rate. In patients with previous history of preterm birth, supplementation is also controversial.

In conclusion, even if the DHA requirements are increased in pregnancy and if positive correlations are reported between low maternal concentrations and an adverse pregnancy outcome, there are conflicting results on the efficacy of a DHA supplementation. Further studies using different DHA regimens of administration are needed. In the meantime, as it is a safe supplementation with few side effects, DHA should be prescribed in patients with a low dietary intake and risks factors.
\end{abstract}

\section{Introduction}

Long chain polyunsaturated fatty acids (LC-PUFA) especially, docosahexaenoic acid (DHA) are important components of the cell membranes integrity and function. During pregnancy and during postpartum, they are essential precursors for prostaglandins and they have been reported to play a role in fetal cognitive and visual development [1,2]. During pregnancy, DHA requirements are increased mainly during the last trimester [3]. Maternal DHA concentration is physiologically reduced by $50 \%$, possibly due to a decreased intake but mainly because of the increased maternal blood volume and enhanced placental and fetal requirements [4]. As fetal LC-PUFA synthesis is limited, its concentration depends mainly on placental transfer. Mechanism of placental LC-PUFA uptake from maternal circulation and transport is not clear but involves binding proteins and placental enzymes. The placental transfer has a high variability as shown in cord DHA levels compared to maternal DHA status. This can be explained by the intake regimens, the duration of DHA supplementation, life style, maternal BMI and placental disorders for a small proportion [5]. The maternal to fetal DHA transfer is facilitated by placental lipases and fatty acid binding proteins [6]. As the need for DHA is increased during the third trimester, a daily supplementation could be recommended [7]. Many studies have reported the benefits of an adequate DHA status during pregnancy and lactation $[8,9]$. The clinical importance of such effects on infant health has not been fully elucidated and is controversial. Therefore, there is a consensus statement for a minimal daily intake of at least $200 \mathrm{mg}$ DHA/day [3]. Generally, dietary advices for consuming food rich in DHA such as oily fish should increase DHA intake and achieve the recommended daily intake [10]. Some supplementation trials, however, used high doses of DHA and reached a higher daily intake of $3 g$ [11].

The aim of this current review is to clarify the relationship between DHA status and pregnancy outcomes regarding mainly the maternal and fetal complications. Indeed, DHA is not only involved in cell integrity and prostaglandin balance but also in the modulation of inflammatory and vascular effects $[12,13]$. Medical evidence-based recommendations are necessary before supporting a systematic supplementation during pregnancy. Controversial data are discussed for each abnormal outcome in this paper.

\section{Methods}

The chosen approach was a systematic search on PubMed using following keywords: DHA and pregnancy; docosahexaenoic acid supplementation and pregnancy.

References published till 2016 including randomized controlled trials (RCT), observational studies and review papers were taken into account.

Correspondence to: Corinne Hubinont, Saint Luc University Hospital, 10 avenue Hippocrate, B-1200 Brussels, Belgium; Tel: +3227641014; Fax: +3227648913; E-mail: corinne.hubinont@uclouvain.be

Key words: DHA, pregnancy, postpartum depression, epilepsy, intrauterine growth restriction, diabetes, obesity, preeclampsia and preterm birth

Received: May 04, 2017; Accepted: May 25, 2017; Published: May 29, 2017 
We evaluated pregnancy outcomes, following specific keywords entered in PubMed in combination with DHA, omega-3, fish oil and LC-PUFA supplementation:

- Maternal outcome: preeclampsia; preterm rupture of membranes (PROM); preterm labor; pregnancy induced hypertension; gestational diabetes; obesity; postpartum depression, prenatal stress.

- Fetal outcome: intrauterine growth restriction (retardation); congenital malformations or anomalies; birth defects

\section{Results}

\section{Meeting the recommended intake}

As the benefits of DHA during pregnancy are not well known by pregnant patients, the consumption of oily fish could be reduced because of the heavy metals related toxicity. Studies showed the importance to develop nutritional education for increasing DHA consumption during pregnancy [14].

In order to evaluate DHA intake during pregnancy, many intervention trials have been conducted. In the Alberta Pregnancy Outcomes and Nutrition (APrON) study including 600 women less than 26 weeks pregnant, the authors found that seafood, fish and seaweed products contributed to $79 \%$ of overall n-3 LCPUFA intake. The study also demonstrated that a DHA supplementation (approximately 275 mg during pregnancy and $299 \mathrm{mg}$ at 3 months postpartum) improved significantly chances of meeting daily recommendations [15].

In terms of supplementation, a daily intake up to $3 \mathrm{~g} /$ day during pregnancy has been demonstrated to be safe [11].

A summary of different recommendation of dietary intakes for DHA is reported in Table $1[16,17]$.

\section{DHA and pregnancy outcomes}

It has been suggested that early DHA supplementation in pregnancy could reduce the incidence of deep placental disorders. The failure of a normal placentation may generate uteroplacental ischemia responsible for several complications including preeclampsia, fetal growth restriction, preterm labor, $\mathrm{PROM}, . .$. In a paper reviewing the effects of DHA supplementation on the placentation disorders prevention, an early first trimester supplementation was associated with a significantly better outcome [18].

Indeed, in vitro, DHA has been shown to stimulate angiogenesis in placental cells. It enhances the expression of major angiogenic factors but also increases the genic expression of intracellular fatty acidbinding proteins (FABP) [19].

A preliminary report compared a group of 28 pregnant women with a DHA supplementation $(300 \mathrm{mg}$ ) after 20 weeks to 50 pregnant controls with no supplementation. There was an association between

Table 1. Recommended dietary DHA supplementation

\begin{tabular}{|c|c|c|c|}
\hline \multirow{2}{*}{ Authority } & \multirow{2}{*}{ Year } & \multicolumn{2}{|c|}{ EPA + DHA $(\mathrm{mg} / \mathrm{d})$ for } \\
\hline & & Adults & Pregnancy \\
\hline $\begin{array}{l}\text { European Food Safety Authority } \\
\text { (EFSA) }\end{array}$ & 2012 & 250 & $\begin{array}{c}250 \text { (of which } 100-200 \\
\text { DHA) }\end{array}$ \\
\hline $\begin{array}{c}\text { Nordic Nutrition } \\
\text { Recommendations (NNR) }\end{array}$ & 2012 & 200-250 DHA & 200 mg DHA \\
\hline WHO & 2003 & $200-1000 / \mathrm{wk}$ & - \\
\hline $\begin{array}{l}\text { National Health and Medical } \\
\text { Research Council (NHMRC) }\end{array}$ & 2006 & $430-610$ & 115 \\
\hline
\end{tabular}

DHA supplementation and low level of placental apoptosis and subsequently with a decreased incidence of placental failure [20,21].

\section{Preeclampsia (PE) and pregnancy induced hypertension}

Preeclampsia is a severe obstetrical complication related to a multifactorial placental dysfunction combining excessive oxidative stress and endothelial dysfunction. Vascular endothelial growth factor (VEGF) is an angiogenic factor involved in preeclampsia: low serum level of VEGF has been reported in the preeclamptic patients compared with that in normal pregnant subjects. VEGF deficit could lead to endothelium cell dysfunction with a possible decreased transfer of fatty acids. Patients with preeclampsia showed a reduced concentration of LC-PUFA and consequently, had a deficiency of anti-inflammatory factors such as lipoxin and prostaglandins [22]. A prospective study comparing preeclamptic women and healthy controls showed also that preeclampsia was associated with a decreased maternal level of plasmatic total omega-3 fatty acids and with a higher level of oxidative stress activity [20]. Wadhwani et al measured serum fatty acid concentrations in 140 normotensive and $54 \mathrm{PE}$ women from the 16th week of gestation till delivery. They found lower levels of DHA in maternal plasma, cord plasma and placenta in the group with PE. They suggested that supplementation of omega- 3 fatty acids during the 16$20^{\text {th }}$ week of gestation could improve fatty acid status in infants born to mothers with PE [23].

Similarly, in another study comparing preeclamptic and control women, a significantly lower maternal and placental DHA level was reported. It was inversely correlated with the seric concentration of an antagonist of VEGF and placental growth factor, the soluble like tyrosine kinase receptor (sFlt-1). The authors suggested that an abnormal LCPUFA metabolism in the membrane with low DHA concentration was responsible for an increased release of sFlt- 1 in the maternal circulation [24]. A follow up study from the same group indicated that low DHA levels were also related to baby gender in women with preeclampsia. They found lower maternal plasma DHA levels in women with preeclampsia delivering male babies as compared to normotensive control women delivering male babies but this difference was not significant in women delivering female babies. This study could have implications in early life if the correlation between DHA maternal blood concentration and neonatal outcome if confirmed [25].

These results are controversial, as a Cochrane Database Systematic Review did not find enough evidence to support the marine oil supplementation in order to reduce the rate of preeclampsia [26]. Of note, this review only included a limited number of studies and refers to ongoing studies, which are also included in this review $[27,28]$.

\section{DHA and obesity}

Obesity during pregnancy is a concern for the obstetrician because of the associated maternal and fetal morbidity [29]. A recent review paper studied the role of n-3 LC-PUFAs in pregnant obese women with metabolic syndrome (MS) .The authors concluded that PUFAs supplementation was not able to prevent preeclampsia, gestational hypertension or gestational diabetes in this obese population. However, they found other benefits such as prevention of preterm delivery and improvement of fetal and neonatal outcome (allergies and neurocognitive performance after birth). They concluded that a supplementation with PUFAs is recommended, although the optimal dose and treatment strategy should be determined [30].

However, in the randomized double-blinded controlled trial called DOMInO (DHA to Optimize Mother Infant Outcome) comparing 
a $800 \mathrm{mg} / \mathrm{d}$ DHA supplementation versus placebo in 2399 pregnant women, there was no change for gestational diabetes nor preeclampsia prevalence in the obese pregnant patients with supplementation [31].

Another double blind RCT studying the effect of omega 3 fatty acids supplementation during pregnancy, showed that the placenta of supplemented obese women was less able to esterify and store lipids, while both birth weight and length were significantly higher than in the control group [32].

\section{DHA and gestational diabetes}

Gestational diabetes mellitus (GDM) is a common pregnancy complication with an increasing prevalence between 3 and $10 \%$. GDM is associated with both short and long term maternal and fetal morbidity. The effect of DHA supplementation on GDM incidence and severity has been previously studied. The DOMInO trial showed that a daily supplementation of $800 \mathrm{mg}$ during the second trimester was not able to reduce the risk of GDM. However, there was a significant effect on perinatal mortality and neonatal convulsions in the DHA treated group [31]. Another RCT using a supplementation of $600 \mathrm{mg}$ reported increased maternal but not fetal DHA levels in pregnant patients with GDM [33]. This finding could be explained by an impaired DHA transfer across the placenta due to GDM as reported by Pagan et al using stable isotope tracers for dynamic studies in vivo [34]. A prospective study on maternal fatty acids concentrations during the third trimester also showed that they were lower in patients with GDM. It could be due to an impaired metabolism instead of an abnormal intake [35].

In conclusion, DHA concentrations are decreased in women with GDM but there is no evidence of GDM prevention in patients receiving DHA supplementation [30,31].

\section{DHA and postpartum depression}

Major depression has been correlated with reduced serum PUFA concentration [36]. The physiological PUFA depletion associated with the last trimester of pregnancy could be responsible for an increased incidence of postpartum depression.

A retrospective observational study comparing women with postpartum depression and controls confirmed the lower serum PUFA concentration in the postpartum depression group and suggested the benefit of a supplementation [37]. Another study reported that the increased risk of postpartum depression was reduced in the presence of a decreased ratio of DHA / n-6 docosapentaenoic acid [38].

However, the DOMInO trial could not evidence a significant difference in the maternal postpartum depression rate between the supplemented and the non supplemented group [39].

In an observational study using a multivariable logistic regression adjusting for social class and maternal age, a weak positive correlation (OR=1.08; 95\% CI:0.98-1.19) was found between DHA levels and depression during pregnancy and postpartum [40].

A 2013 Cochrane Database Systematic Review aiming to assess the benefits of dietary supplements (Selenium, DHA, EPA...) for preventing postpartum depression included two RCTs using different daily DHA intake ( $900 \mathrm{mg}$ and $274 \mathrm{mg}$ ) versus placebo. There was no difference of postpartum depression prevalence in the two groups. The authors could not conclude on the evidence-based effect of DHA supplementation on postnatal depression and could not recommend it as prevention [41].
Another study based on 900 pregnant patients compared the blood fatty acids (DHA, EPA, omega 3 ) status with a standardized evaluation of postnatal depression. There was no correlation between the serum PUFA concentrations and the postpartum depression gravity criteria [42]. In conclusion, apart one study, there is no evidence that a DHA supplementation may prevent postpartum depression.

\section{DHA and prenatal stress}

Prenatal stress is a well-known multifactorial pathology affecting the outcome of the pregnancy and the offspring both in human and animal models $[43,44]$. In a study performed in non-pregnant patients, PUFA supplementation has been shown to reduce stress-induced effects on appetite, mood, mental concentration, and sleep quality. This positive action was also correlated with seric cortisol levels [45]. In order to confirm an effect on prenatal stress, an investigation was initiated in low-income African American pregnant women enrolled between 16 and 21 weeks and randomized with either a $450 \mathrm{mg} / \mathrm{d}$ DHA supplementation or placebo. The supplemented group was found to have a reduced stress scale quote and lower seric cortisol concentrations. This preliminary study supports the use of a DHA supplementation for improving both maternal and fetal outcomes in pregnant patients at risk for prenatal stress [46].

\section{DHA and fetal growth}

Fetal growth requires the presence of a normal placental function and an adequate maternal nutritional status. Low birth weight (LBW) is associated with short and long term morbidity and mortality. Several studies reported the effect of DHA supplementation on newborn weight.

A small RCT including 43 women at high risk for preterm delivery tested the effect of vaginally administered DHA versus placebo. The supplemented group had a significant increase in gestational age at delivery and in newborn birth weight [47].

In a prospective study, Wadhwani et al reported a positive correlation between the maternal and umbilical cord plasma DHA concentrations and birth weight [48].

In a double blind RCT including 350 women receiving either $600 \mathrm{mg} / \mathrm{d}$ DHA or placebo, the authors found that supplementation during the last half of gestation resulted in overall greater gestation duration and infant size [8].

However, another double-blind (400 mg DHA versus placebo) RCT, based on 1094 pregnant women, did not show any difference in the mean gestational age, weight and head circumference between the 2 groups. When data were analysed according to gravidity, the primigravidae offspring were significantly heavier and had larger head circumferences than controls [27]. All studies but one support a positive effect of DHA supplementation on birth weight and fetal growth.

\section{DHA and fetal intrauterine growth restriction}

Intrauterine growth restriction (IUGR) is a multifactorial disorder. When it is associated to placental insufficiency, fetal lipid metabolism may be dysfunctional. Studies on animal models reported an impaired DHA transplacental transfer in IUGR. This could be partly responsible for the neurological sequellae associated with this pathology [6].

In a prospective study on pregnant women, the first trimester DHA concentration in maternal erythrocytes was significantly correlated 
with neonatal weight. DHA levels were lower in women delivering low birth weight infants compared to controls [49].

However, the role of DHA prevention is controversial: a Cochrane database systematic review did not evidence a reduced prevalence of IUGR when a supplementation by marine oil was prescribed [26].

A recent systematic review based on three RCTs evaluating the effects of omega -3 supplementation in patients with a previous IUGR, did not find any significant difference in birth weight between the supplemented patients and controls. The incidence of perinatal death was similar in both groups. This review did not support a routine DHA supplementation for preventing recurrent IUGR [50].

\section{DHA and Prevention of preterm birth (PTB) and Premature rupture of membranes (PROM)}

The role of DHA in the prevention of PROM and preterm labor has been studied and remains controversial: as preterm labour is a multifactorial process, it is difficult to compare outcomes associated with different causes. Inflammation is one of the leading causes of PTB and PROM [51].

In general inflammation process is mediated by cytokines and prostaglandins. A study established that TNF $\alpha$ and interleukin release could be down regulated by a diet with fish oil suggesting a protective effect against inflammation consequences [52]. A possible effect of DHA supplementation on the risk of PROM, placental inflammation and oxidative stress incidence has been reported in a pilot study in healthy pregnant women [7]. Oxidative stress could affect the secretion of neurotrophic factors such as the brain-derived neurotrophic factor and nerve growth factor, proteins involved in both angiogenesis and placental development. In preterm delivery, their concentration are decreased specially in patients with abnormal omega 3 fatty acids levels [53].

In a systematic review and meta-analysis published in 2010, the benefits of n-3 fatty acids were clearly demonstrated: women with n-3 fatty acids supplementation delivered significantly later compared to the control group. The mean birth weight of the first group was also higher [54].

An expert workshop of the Early Nutrition Academy concluded that an additional supply of $200 \mathrm{mg} /$ day of DHA in order to reach a total intake of $300 \mathrm{mg}$ during pregnancy, is beneficial for preventing preterm labour before 34 weeks [55].

An observational study in women consuming fish and receiving an additional supplementation of $200 \mathrm{mg}$ DHA /day showed that they have a lower incidence of preterm labour and an increased neonatal weight compared to controls without supplementation [56].

However, a Cochrane database systematic review did not find enough evidence to recommend a marine oil supplementation in order to reduce the rate of preterm birth [26].

A more recent systematic review and meta-analysis of 9 RCTs on the preventive role of omega-3 supplementation in asymptomatic singleton pregnancies with a previous history of preterm labour, was not conclusive for a beneficial effect on the outcome. Supplemented group and controls had similar incidence of preterm labour and secondary neonatal outcome. However, in the omega- 3 treated group, there was a significant longer latency and higher birth weight suggesting a possible benefit of the supplementation [57].

\section{DHA and other fetal disorders}

The cause for congenital malformations is multifactorial. Many studies have reported the role of a deficient diet during conception and pregnancy on congenital abnormality incidence. For instance, a Cochrane database systematic review strongly recommends a systematic supplementation of folic acid to prevent neural tube defects [58].

The role of PUFA in fetal development and subsequently on birth defects is not clear. A pilot case study based on pregnant patients with fetuses affected by gastroschisis. The lipids pattern was evaluated in maternal and neonatal blood: there was a significantly higher DHA concentration-, in gastroschisis mothers at delivery compared to controls. Moreover, the association of low oleic acid, low lipogenic index and increased desaturation index in gastroschisis mothers, suggests that the imbalance in fatty acids associated with either a vascular or an inflammatory process may be the underlying mechanism responsible for this birth defect [59].

Zellweger syndrome is a congenital disorder associated with dysfunctional peroxisomes, intracellular organelles involved in lipids oxydation and biosynthesis. Patients affected by the syndrome were found to have low DHA concentration in the brain and other organs .The effect of a DHA supplementation (100-500 mg per day) improved the DHA levels, the liver function as well as the vision [60]. There is no longitudinal study on the effect of a prenatal supplementation and the Zellweger incidence.

Autism is a multifactorial process associated with genetic and environmental causes. Some forms of the disorder have been linked to altered PUFA metabolism. Micronutrients deficiency plays an important role on neurotrophic factors and neurotransmitters synthesis and effects. Even if the mechanism remains unclear, adverse events occurring prenatally such as malnutrition could also induce an excessive inflammatory response partly responsible for brain disorders [61].

However, the protective effect of a prenatal DHA supplementation on autism incidence has not been reported yet.

\section{DHA and perinatal mortality}

Either related or independent of the neonatal morbidity due to maternal or fetal disorders, perinatal mortality remains a concern. In a metanalysis reviewing 34 RCTs, the authors found in singleton pregnancies supplemented from the second trimester a significant reduction in the perinatal mortality rate. Further studies for confirming this data are required [57].

\section{Discussion}

DHA is a key nutrient for the metabolism, especially during pregnancy and lactation. The benefits of DHA are based on its antiinflammatory and anti-oxidative properties improving both placental blood flow and amniotic membranes resistance. It is well established that during the last trimester of pregnancy, maternal seric DHA concentrations are decreased not only because of vascular volume extension but also by maternal, placental and fetal DHA increased requirements $[1,2,11]$. There are some physiological adaptation mechanisms such as DHA synthesis from fatty acids but a diet with oily fish for achieving the minimum intake is strongly recommended [10].

Several regimens of DHA supplementation have been evaluated in RCT and observational studies. If the effect of supplementation remains controversial, at least it has been proved to be safe $[11,16,17]$. Part of the explanation of this controversy is the discrepancy in cord 
blood concentrations: the difference in gestational age and in placental transfer function could be responsible for it [5]. Another explanation could be the lack of control on the quality of DHA supplements used in the studies, as LC-PUFA could be more or less oxidated when administered. It is not well established whether this parameter affects both efficacy and safety [62].

How and when should DHA supplementation be recommended and in which form?

There's no clear answer to this question yet. The Word Health Organization (WHO) states that current evidence on supplementation with n-3 LC-PUFA is indicative for a reduced risk on preterm delivery, however they still need more research before they will make specific recommendations [63]. EFSA allows reaching the Dietary Reference Value for LC-PUFA (250 mg EPA plus DHA, plus and additional 100$200 \mathrm{mg}$ of DHA) either by food and supplements [64].

The authorities do not recommend a specific form for supplementation. A recent North American study compares several fish oil supplements on the market. The authors concludes that highly concentrated fish oil in unflavored soft gel capsules seems the best option to prevent the fragile n-3 LC-PUFA from deteriorating oxidation. Quality standards should be established by medication regulation instances in order to guarantee the best efficacy of fish oil [62].

What is the effect of a supplementation on maternal and fetal outcome?

A recent systematic review of Level -1 data and based on 34 RCTs aiming to establish recommendations for DHA supplementation during pregnancy was unable to provide evidence for a preventive effect pregnancy complications. However, the authors reported a positive effect of supplementation on neonatal outcome of patients with gestational diabetes (hyperbilirubinemia rate, hospitalization rate) as well as a significant reduction in perinatal death in singleton pregnancies supplemented before 20 weeks of gestation [65].

Our review paper aims to analyse the controversies found in the literature. Some papers report an improved outcome for preventing placentation disorders [18]. But 3 Cochrane database systematic reviews and recent meta-analysis are not conclusive and do not recommend a DHA supplementation for the prevention of preeclampsia, gestational diabetes and postpartum depression [26,30,31,41].

DHA supplementation reduces significantly the effects of prenatal stress and the perinatal mortality rate and also, increases the birth weight $[46,48]$. However for recurrent IUGR, it is not clear that a routine supplementation could improve fetal growth [50]. In patients with previous history of premature rupture of the membranes and preterm birth, the preventive effect of a supplementation was found in some studies [54-56] but it is controversial as a recent meta-analysis does not support a beneficial effect of DHA on preterm labour incidence [57].

\section{Conclusion}

The role of LC-PUFA, especially DHA for the materno-fetal and placental physiology is well known. Pregnancy is associated with an increased need for DHA and a possible depletion of DHA in the maternal stores. Many studies reported a correlation between low serum levels of PUFA and adverse outcomes in pregnancy. An increased dietary fat intake should be recommended during pregnancy. There are conflicting results on the efficacy of a DHA supplementation. A recent meta-analysis does not support a preventive effect of DHA supplementation on some pregnancy complications but other studies report however a significant effect in the presence of prenatal stress and on the perinatal mortality rate and birth weight. A better knowledge of transplacental transfer, of polymorphisms on fatty acid metabolism enzymes genes and also the galenic properties of supplementation could explain the controversial results found in the literature. In the meantime, DHA should be prescribed in patients with a low dietary intake and risks factors, as it is a safe supplementation with few side effects.

\section{Declaration of interest statement}

TS works as a freelance medical affair consultant for Merck Consumer Healthcare Belgium.

\section{References}

1. Scholtz SA, Colombo J, Carlson SE. (2013) Clinical Overview of Effects of Dietary LCPUFA during the Perinatal Period. Nestle Nutr Inst Workshop Ser 77:145-154. [Crossref]

2. Al M, van Houwelingen A, Hornstra G. (2000) Long-chain polyunsaturated fatty acids, pregnancy, and pregnancy outcome. Am J Clin Nutr. 71(Suppl 1):285S-91S.

3. Koletzko B, Cetin I, Brenna JT et al. (2007) Dietary fat intakes for pregnant and lactating women. Br J Nutr 98: 873-877. [Crossref]

4. Holman RT, Ogburn PL. Johnson SB (1991) Deficiency of essential fatty acids and membrane fluidity during pregnancy and lactation. Proc Natl Acad Sci USA. 88:48354839. [Crossref]

5. Muhlhausler B, Gibson R, Yelland L, Makrides M. (2014) Heterogenity in cord blood DHA concentration: towards an explanation. Prostaglandins Leuko Essent Fatty Acids. 91:135-140. [Crossref]

6. Hanebutt FL1, Demmelmair H, Schiessl B, Larqué E, Koletzko B (2008) Long-chain polyunsaturated fatty acid (LC-PUFA) transfer across the placenta. Clin Nutr 27: 685693. [Crossref]

7. Pietrantoni E, Del Chierico F, Rigon G, Vernocchi P, Salvatori G et al. (2014) Docosahexaenoic acid supplementation during pregnancy: a potential tool to preven membrane rupture and preterm labor. Int J Mol Sci. 15:8024-8036.

8. Carlson SE, Colombo J, Gajewski BJ, Gustafson KM, Mundy D et al. (2013) DHA supplementation and pregnancy outcomes. Am J Clin Nutr 97: 808-815. [Crossref]

9. Ramakrishnan U, Imhoff-Kunsch B, Martorell R (2014) Maternal nutrition interventions to improve maternal, newborn, and child health outcomes. Nestle Nutr Inst Workshop Ser 78: 71-80. [Crossref]

10. Miles E, Noakes P, Kremmyda L, Vlachava M, Diaper N Rosenlund G, Urwin H Yaqoob P, Rossary A, Farges M, et al. (2011) The Salmon in Pregnancy Study: study design, subject characteristics, maternal fish and marine n-3 fatty acid intake, and marine n-3 fatty acid status in maternal and umbilical cord blood. Am J Clin Nutr. 94:1986S-1992S. [Crossref]

11. Makrides M. (2009) Is there a dietary requirement for DHA in pregnancy? Prostaglandins Leukot Essent Fatty Acids. 81:171-174. [Crossref]

12. Ruan D, So S. (2012) Screening and identification of dietary oils and unsaturated fatty acids in inhibiting inflammatory prostaglandin E2 signaling in fat stromal cells. BMC Complementary and Alternative Medicine. 12:143. [Crossref]

13. Lorente-Cebrián S1, Costa AG, Navas-Carretero S, Zabala M, Laiglesia LM, et al (2015) An update on the role of omega-3 fatty acids on inflammatory and degenerative diseases. J Physiol Biochem 71: 341-349. [Crossref]

14. Emmett R1, Akkersdyk S, Yeatman H, Meyer BJ (2013) Expanding awareness of docosahexaenoic acid during pregnancy. Nutrients 5: 1098-1109. [Crossref]

15. Jia X, Pakseresht M, Wattar N, Wildgrube J, Sontag S et al. (2015) Women who take $\mathrm{n}-3$ long-chain polyunsaturated fatty acid supplements during pregnancy and lactation meet the recommended intake. Appl Physiol Nutr Metab. 40:474-481. [Crossref]

16. EFSA Panel on Dietetic Products, Nutrition and Allergies (NDA). Scientific Opinion related to the Tolerable Upper Intake Level of eicosapentaenoic acid (EPA) docosahexaenoic acid (DHA) and docosapentaenoic acid (DPA). EFSA Journal. 2012;10(7):2815.

17. Nordic Council of Ministers. Nordic Nutrition Recommendation. [Internet]. 2012 [cited 2017 Apr 17]. Available from: https://www.norden.org/en/theme/nordic-nutritionrecommendation/nordic-nutrition-recommendations-2012. 
18. Carvajal J (2014) Docosahexaenoic acid supplementation early in pregnancy may prevent deep placentation disorders. Biomed Res Int. 2014:526895. [Crossref]

19. Basak S, Das M, Duttaroy A (2013) Fatty acid-induced angiogenesis in first trimester placental trophoblast cells: possible roles of cellular fatty acid-binding proteins. Life Sci. 93(21):755-62. [Crossref]

20. Mehendale S, Kilari A, Dangat K, Taralekar V, Mahadik S et al. (2008) Fatty acids, antioxidants, and oxidative stress in pre-eclampsia. Int J Gynaecol Obstet 100: 234238. [Crossref]

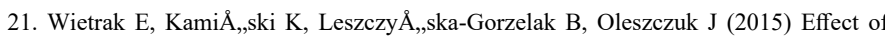
Docosahexaenoic Acid on Apoptosis and Proliferation in the Placenta: Preliminary Report. Biomed Res Int 2015: 482875. [Crossref]

22. Das UN1 (2015) Cytokines, angiogenic, and antiangiogenic factors and bioactive lipids in preeclampsia. Nutrition 31: 1083-1095. [Crossref]

23. Wadhwani N (2014) Altered maternal proportions of long chain polyunsaturated fatty acids and their transport leads to disturbed fetal stores in preeclampsia. Prostaglandins Leukot Essent Faaty Acids. 91: 21-30. [Crossref]

24. Kulkarni A, Mehendale S, Yadav H, Joshi S (2011) Reduced placental docosahexaenoic acid levels associated with increased levels of sFlt-1 in preeclampsia. Prostaglandins, Leukotrienes and Essential Fatty Acids. 84:51-55. [Crossref]

25. Roy S, Dhobale M, Dangat K, Mehendale S, Wagh G et al. (2014) Differential levels of long chain polyunsaturated fatty acids in women with preeclampsia delivering male and female babies. Prostaglandins Leukot Essent Fatty Acids. 91(5):227-32. [Crossref]

26. Makrides M, Duley L, Olsen SF (2006) Marine oil, and other prostaglandin precursor, supplementation for pregnancy uncomplicated by pre-eclampsia or intrauterine growth restriction. Cochrane Database Syst Rev : CD003402. [Crossref]

27. Ramakrishnan U, Stein AD, Parra-Cabrera S, Wang M, Imhoff-Kunsch B et al. (2010) Effects of docosahexaenoic acid supplementation during pregnancy on gestational age and size at birth: randomized, double-blind, placebo-controlled trial in Mexico. Food Nutr Bull 31: S108-116. [Crossref]

28. Ramakrishnan U, Stinger A, DiGirolamo A, Martorell R, Neufeld L et al. (2015) Prenatal Docosahexanoic Acid Supplementation and Offspring Development at 18 Months: Randomized Controlled Trial. PLoS one 11:e0120065. [Crossref]

29. Zambrano E, Ibáñez C, Martínez-Samayoa PM, Lomas-Soria C, Durand-Carbajal M et al. (2016) Maternal Obesity: Lifelong Metabolic Outcomes for Offspring from Poor Developmental Trajectories During the Perinatal Period. Arch Med Res 47: 1-12. [Crossref]

30. Poniedzialek-Czajkowska E, Mierzynski R, Kimber-Trojnar Z, Leszczynska-Gorzelak B, Oleszczuk J. (2014) Polyunsaturated fatty acids in pregnancy and metabolic syndrome: a review. Curr Pharm Biotechnol. 15(1):84-99. [Crossref]

31. Zhou SJ, Yelland L, McPhee AJ, Quinlivan J, Gibson RA, et al. (2012) Fish-oil supplementation in pregnancy does not reduce the risk of gestational diabetes or preeclampsia. Am J Clin Nutr 95: 1378-1384. [Crossref]

32. Calabuig-Navarro V, Puchowicz M, Glazebrook P, Haghiac M, Minium J, et al. (2016) Effect of w-3 supplementation on placental lipid metabolism in overweight and obese women. Am J Clin Nutr 103: 1064-1072. [Crossref]

33. Min Y, Djahanbakhch O, Hutchinson J, Eram S, Bhullar A et al. (2016) Efficacy of docosahexaenoic acid-enriched formula to enhance maternal and fetal blood docosahexaenoic acid levels: Randomized double-blinded placebo-controlled trial of pregnant women with gestational diabetes mellitus. Clin Nutr. 35:608-14. [Crossref]

34. Pagan A, Prieto-Sánchez M, Blanco-Carnero J, Gil-Sánchez A, Parrilla J et al. (2013) Materno-fetal transfer of docosahexaenoic acid is impaired by gestational diabetes mellitus. Am J Physiol Endocrinol Metab. 305:E826-33. [Crossref]

35. Zhao JP, Levy E, Shatenstein B, Fraser WD et al. (2016) Longitudinal circulating concentrations of long-chain polyunsaturated fatty acids in the third trimester of pregnancy in gestational diabetes. Diabet Med 33: 939-946. [Crossref]

36. Adams P, Lawson S, Sanigorski ASA. (1996) Arachidonic acid to eicosapentaenoic acid ratio in blood correlates positively with clinical symptoms of depression. Lipids. 31:S157-61. [Crossref]

37. De Vriese S, Christophe A, Maes M. (2003) Lowered serum n-3 polyunsaturated fatty acid (PUFA) levels predict the occurrence of postpartum depression: further evidence that lowered n- PUFAs are related to major depression. Life Sci. 73:3181-7. [Crossref]

38. Otto SJ, de Groot RH, Hornstra G (2003) Increased risk of postpartum depressive symptoms is associated with slower normalization after pregnancy of the functional docosahexaenoic acid status. Prostaglandins Leukot Essent Fatty Acids 69: 237-243. [Crossref]
39. Makrides M, Gibson R, McPhee A, Yelland L, Quinlivan J et al. (2010) Effect of DHA Supplementation During Pregnancy on Maternal Depression and Neurodevelopment of Young Children. JAMA. 304: 1675-83. [Crossref]

40. Sallis H, Steer C, Paternoster L, Davey Smith G, Evans J (2014) Perinatal depression and omega-3 fatty acids: a Mendelian randomisation study. $J$ Affect Disord 166: 124131. [Crossref]

41. Miller B, Murray L, Beckmann M, Kent T (2013) Macfarlane B Dietary supplement for preventing postnatal depression. Cochrane Database Syst Rev. 10:CD009104. [Crossref]

42. Parker G, Hegarty B, Granville-Smith I, Ho J, Paterson A et al. (2015) Is essential fatty acid status in late pregnancy predictive of post-natal depression? Acta Psychiatr Scand 131: 148-156. [Crossref]

43. Henry C, Kabbaj M, Simon H, Le Moal M, Maccari S (1994) Prenatal stress increases the hypothalamo-pituitary-adrenal axis response in young and adult rats. $J$ Neuroendocrinol. 6: 341-5. [Crossref]

44. King S, Dancause K, Turcotte-Tremblay AM, Veru F, Laplante DP (2012) Using natural disasters to study the effects of prenatal maternal stress on child health and development. Birth Defects Res C Embryo Today. 96:273-88. [Crossref]

45. Yehuda S, Rabinovitz S, Mostofsky DI (2005) Mixture of essential fatty acids lowers test anxiety. Nutr Neurosci 8: 265-267. [Crossref]

46. Keenan K, Hipwell AE, Bortner J, Hoffmann A, McAloon R (2014) Association between fatty acid supplementation and prenatal stress in African Americans: a randomized controlled trial. Obstet Gynecol 124: 1080-1087. [Crossref]

47. Giorlandino C, Giannarelli D (2013) Effect of vaginally administered DHA fatty acids on pregnancy outcome in high risk pregnancies for preterm delivery: a double blinded randomised controlled trial. J Prenat Med 7: 42-45. [Crossref]

48. Wadhwani N, Pisal H, Mehendale S, Joshi S. A prospective study of maternal fatty acid, micronutrients and homcysteine and their association with birth outcome. Matern Child Nutr. 2015 Jun 25;11(4):559-73. [Crossref]

49. Meher A, Randhir K, Mehendale S, Wagh G, Joshi S (2016) Maternal Fatty Acids and Their Association with Birth Outcome: A Prospective Study. PLoS One 11: e0147359. [Crossref]

50. Saccone G, Berghella V, Maruotti GM, Sarno L, Martinelli P. (2015) Omega-3 supplementation during pregnancy to prevent recurrent intrauterine growth restriction: systematic review and meta-analysis of randomized controlled trials. Ultrasound Obstet Gynecol.;46:659-664. [Crossref]

51. Cappelletti M, Della Bella S, Ferrazzi E, Mavilio D, Divanovic S (2016) Inflammation and preterm birth. J Leukoc Biol 99: 67-78. [Crossref]

52. Caughey GE, Mantzioris E, Gibson RA, Cleland LG, James MJ (1996) The effect on human tumor necrosis factor alpha and interleukin 1 beta production of diets enriched in n-3 fatty acids from vegetable oil or fish oil. Am J Clin Nutr 63: 116-122. [Crossref]

53. Dhobale M, Joshi S (2012) Altered maternal micronutrients (folic acid, vitamin B(12)) and omega 3 fatty acids through oxidative stress may reduce neurotrophic factors in preterm pregnancy. J Matern Fetal Neonatal Med 25: 317-323. [Crossref]

54. Salvig J, Lamont R. (2011) Evidence regarding an effect of marine n-3 fatty acids on preterm birth: a systematic review and meta-analysis. Acta Obstet Gynecol Scand. 90:825-838. [Crossref]

55. Koletzko B, Boey C, Campoy C, Carlson S, Chang N et al. (2014) Current Information and Asian Perspectives on Long-Chain Polyunsaturated Fatty Acids in Pregnancy, Lactation, and Infancy: Systematic Review and Practice Recommendations from an Early Nutrition Academy Workshop. Ann Nutr Metab 65(1):49-80. [Crossref]

56. Le Donne M, Alibrandi A, Vita R, Zanghì D, Triolo O, et al. (2016) Does eating oily fish improve gestational and neonatal outcomes? Findings from a Sicilian study Women Birth 29: e50-57. [Crossref]

57. Saccone G, Berghella V (2015) Omega-3 long chain polyunsaturated fatty acids to prevent preterm birth: a systematic review and meta-analysis. Obstet Gynecol 125: 663672. [Crossref]

58. De-Regil LM, Peña-Rosas JP, Fernández-Gaxiola AC, Rayco-Solon P (2015) Effects and safety of periconceptional oral folate supplementation for preventing birth defects. Cochrane Database Syst Rev : CD007950. [Crossref]

59. Jones KL, Weiss LA, Hagey LR, Gonzalez V, Benirschke K et al. (2013) Altered lipid metabolism in gastroschisis: a novel hypothesis. Am J Med Genet A 161A: 1860-1865. [Crossref] 
60. Martinez M (2001) Restoring the DHA levels in the brains of Zellweger patients. J Mol Neurosci 16: 309-316. [Crossref]

61. Das U. (2013) Autism as a disorder of deficiency of brain-derived neurotrophic factor and altered metabolism of polyunsaturated fatty acids. Nutrition 29: 1175-85. [Crossref]

62. Jackowski SA, Alvi AZ, Mirajkar A, Imani Z. (2015) Oxidation levels of North American over-the-counter n-3 (omega-3) supplements and the influence of supplement formulation and delivery form on evaluating oxidative safety. Journal of Nutritional Science4: e30. [Crossref]
63. WHO. Long chain polyunsaturated fatty acid supplementation during pregnancy. [Internet]. 2017 [cited 2017 Mar 19]. Available from: http://www.who.int/elena/titles/ fish_oil_pregnancy/en/.

64. EFSA Panel on Dietetic Products, Nutrition, and Allergies (NDA). (2010) Scientific Opinion on Dietary Reference Values for fats, including saturated fatty acids, polyunsaturated fatty acids, monounsaturated fatty acids, trans fatty acids, and cholesterol. EFSA Journal 8(3):1461.

65. Saccone G, Saccone I, Berghella V. (2016) Omega-3 long-chain polyunsaturated fatty acids and fish oil supplementation during pregnancy: which evidence? J Matern Fetal Neonatal Med 29: 2389-2397. [Crossref]

Copyright: (C2017 Hubinont C. This is an open-access article distributed under the terms of the Creative Commons Attribution License, which permits unrestricted use, distribution, and reproduction in any medium, provided the original author and source are credited. 